\title{
Intraosseous Access for Neonatal and Newborn Resuscitation in the National Park Service (NPS)
}

\author{
Eric R. Schmitt, MD, MPH; ${ }^{1}$ Geoff Stroh, MD, FACEP, NPS EMS Medical Advisor; ${ }^{2}$ \\ Marc Shalit, MD, FACEP, NPS EMS Medical Advisor; ${ }^{2}$ \\ Danielle Campagne, MD, NPS EMS Medical Advisor ${ }^{2}$
}

1. Harbor-UCLA Medical Center, Department of Emergency Medicine, Torrance, California USA

2. UCSF-Fresno Medical Education Program, Fresno, California USA

Correspondence:

Geoff Stroh, MD

155 N. Fresno Street Suite 206

Fresno, California USA 93701

\author{
Abbreviations: \\ ALS = advanced life support \\ $\mathrm{IO}=$ intraosseous \\ $\mathrm{IV}=$ intravenous \\ UVS = umbilical vein catheter
}

doi:10.1017/S1049023X11006285
The primary providers of advanced life support (ALS) medical care in national parks are specially trained rangers, known as "Parkmedics." These providers are trained to an Emergency Medical Technician-Intermediate-ALS level with an expanded scope of practice that includes $>20$-medications, intravenous (IV), and airway skills. Additionally, they are trained to obtain intraosseous (IO) access in children if needed. For newborns, they currently are taught to clamp the cord, and if IV access is indicated, obtain access in an umbilical vein, using standard IV technique through the side of the umbilical cord. We recently sought to determine if IO access should be included in the Parkmedic protocols for neonatal and newborn resuscitation by these providers.

The use of $\mathrm{IO}$ access for pediatric patients in the prehospital setting has been studied and is an accepted standard, but whether this intervention can be extended into the neonatal and especially the newborn ( $<24$ hours) period is not clear. ${ }^{1}$ There are case reports of IO lines being used for neonates in the neonatal intensive care unit (NICU), ${ }^{2-6}$ and in the emergency department. ${ }^{7}$ Intraosseous access in the neonate appears to be safe, and reported complications are limited to rare cases of compartment syndrome from infiltrated lines. ${ }^{8}$ However, most published reports describe older neonates who have had other previous forms of vascular access. The use of IO as a first- or second-line choice for access in the first 24 hours of life is more controversial.

The 2000 International Guidelines for Cardiopulmonary Resuscitation and Emergency Cardiovascular Care state, "Intraosseous lines are not commonly used in newly born infants because of the more readily accessible umbilical vein, the fragility of small bones, and the small intraosseous space in the premature infant." However, they add that, "Intraosseous access can be used as an alternative route for medication/volume expansion if umbilical or other direct venous access is not readily attainable (Class IIb, LOE5)." "The 2005 update of the Guidelines makes no comment on routes of vascular access.

Perhaps the best clinical evidence comes from Ellemunter et al, who presented a case series of neonates who were resuscitated via IO access within five hours of birth. ${ }^{10}$ These 27 (20 pre-term and SEVEN term) critically ill neonates, all received 18-gauge IO lines when other routes of access failed, and the IO was left in place for up to 20 hours. In this series, the providers were experienced, and they reported no failed attempts, major complications, or adverse events.

The ability of a novice provider to replicate this success is unknown, but Abe et al provided some guidance in their study of inexperienced medical students. ${ }^{11} \mathrm{The}$ students made two attempts each at placing IO and umbilical vein catheter (UVC) lines in a simulated neonatal model. Placement of the IO line was faster and subjectively easier than the UVC on both initial and repeat attempts. The authors suggested that "IO results in easier and more rapid vascular access in those who do not frequently perform newborn resuscitation." In a recent review of neonatal IO access, Engle noted that the available literature is limited, but concluded that "Intraosseous access is most likely to be needed in out-of-hospital settings... or when clinicians responsible for vascular access during resuscitations are more skilled with IO access than with IV access."12 
Although limited, it appears that the available literature supports the use of IO access for neonatal and newborn resuscitation, and there is some suggestion that it even may be a preferred route of access for providers with limited experience,

\section{References}

1. Frascone RJ, Jensen J, Wewerka SS, Salzman JG: Use of the pediatric EZ-IO needle by emergency medical services providers. Pediatr Emerg Care 2009;25(5):329-332.

2. Kelsall AW: Resuscitation with intraosseous lines in neonatal units. Arch Dis Child 1993;68(3 Spec No):324-325.

3. Martino Alba R, Ruiz Lopez MJ, Casado Flores J: Use of the intraosseous route in resuscitation in a neonate. Intensive Care Med 1994;20(7):529.

4. Kakhandki SK: Intraosseous infusion in a LBW neonate. Indian Pediatr 1997;34(8):748-749.

5. Ramet J, Clybouw C, Benatar A, Hachimi-Idrissi S, Corne L: Successful use of an intraosseous infusion in an 800 grams preterm infant. Eur J Emerg Med 1998;5(3):327-328

6. Lake W, Emmerson AJ: Use of a butterfly as an intraosseous needle in an oedematous preterm infant. Arch Dis Child Fetal Neonatal Ed 2003;88(5):F409.

7. Friedman FD: Intraosseous adenosine for the termination of supraventricular tachycardia in an infant. Ann Emerg Med 1996;28(3):356-358. or in the prehospital setting. This has led us to endorse the use of IO access as the first line option for vascular access during neonatal and newborn resuscitation in the National Park Service.

8. Burke T, Kehl DK: Intraosseous infusion in infants. Case report of a complication. J Bone Joint Surg Am 1993;75(3):428-429.

9. Contributors and Reviewers for the Neonatal Resuscitation Guidelines: An excerpt from the Guidelines 2000 for Cardiopulmonary Resuscitation and Emergency Cardiovascular Care: International Consensus on Science. Pediatrics 2000; 106(3):e29.

10. Ellemunter H, Simma B, Trawöger R, Maurer H: Intraosseous lines in preterm and full term neonates. Arch Dis Child Fetal Neonatal Ed 1999;80(1):F74-F75.

11. Abe KK, Blum GT, Yamamoto LG: Intraosseous is faster and easier than umbilical venous catheterization in newborn emergency vascular access models. Am J Emerg Med 2000;18(2):126-129.

12. Engle WA: Intraosseous access for administration of medications in neonates. Clin Perinatol 2006;33(1):161-168,ix. 